\title{
Study Serum Level of Interleukin-17 in Pediatric Systemic Lupus Erythematosus
} Doaa Mostafa Tawfik ${ }^{1}$, Mayy Abdel-Fattah Moahmed ${ }^{1}$, Naglaa Ali Khalifa ${ }^{2}$, Asmaa Ibrahim Mohamed Ged ${ }^{1}$

Departments of ${ }^{1}$ Pediatrics and ${ }^{2}$ Clinical Pathology, Faculty of Medicine, Zagazig University, Sharkia, Egypt

*Corresponding Author: Asmaa Ibrahim Mohamed Ged, Mobile: (+20)01005416759, Email: dr.asmaagad2010@ gmail.com

\begin{abstract}
Background: Systemic lupus erythematosus (SLE) is a complex autoimmune disease of unknown etiology. Objective: To assess serum levels of IL-17 in pediatric SLE (pSLE) and their association with clinical manifestations, laboratory findings, SLE disease activity index score (SLEDAI), and therapy.

Patients and Methods: A case-control study was conducted at Pediatric Nephrology units, and Outpatient Nephrology Clinic of Children Hospital, Zagazig University. It was carried out over two years from September 2017 to August 2019. This study was performed on 60 patients classified into two groups: thirty of them were children with SLE and 30 apparently healthy children as a Control group of comparable age and gender. All patients were subjected to complete history taking and clinical examination. Laboratory investigations were done. Renal biopsy was done to 22 cases who were presented by renal symptoms.

Results: Frequency of manifestation were malar rash $86.7 \%$, photosensitivity $80.0 \%$, renal manifestations, and anemia73.3\% for each. SLEDAI score was 18 - 38. Class II lupus nephritis (LN) was the most common biopsies result $(40 \%)$. The range of IL-17 for cases was $(89.3-359 \mathrm{pg} / \mathrm{ml})$, a highly significant increase in the Il-17 level in the case group than the control. A significant association between IL-17 level and renal manifestation, urea, creatinine, 24-hour urinary protein, and SLEDAI.

Conclusions: Serum IL-17A is elevated more than 170 in pSLE patients with lupus nephritis, which correlates with disease activity and renal biopsy. IL-17 seems to have a possible role in the pathogenesis of lupus nephritis.

Keywords: Systemic lupus erythematosus (SLE), interleukin-17 (IL-17), Lupus nephritis (LN), Systemic Lupus Erythematosus Disease Activity Index (SLEDAI).
\end{abstract}

\section{INTRODUCTION}

Systemic lupus erythematosus (SLE) is a complex, multi-system autoimmune disease of unknown etiology. It is characterized by autoantibodies production, immune complex formation and immune dysregulation result in damage to the multisystem include the kidney (1). SLE has a wide range of disease manifestations and can lead to significant morbidity and even mortality ${ }^{(2)}$. Children are at higher risk for severe SLE, typically presenting with higher disease activity and continuing with higher activity over time than adults. Presentation in childhood occurs in 15-20\% of all SLE patients, with median diagnosis age for pSLE at 12 years ${ }^{(3)}$. Children are more likely to have serious renal, neurological, and hematological involvement than their adult counterparts (4). Mortality rates are higher in pSLE patients than adults, despite having less comorbidity ${ }^{(5)}$.

Lupus nephritis (LN) is the most common severe manifestation of SLE. It is a serious complication of SLE, influencing prognosis, and life expectancy more than any other organ system involvement ${ }^{(6)}$. Furthermore, LN is common in children. SLE in children and adolescent has higher rates of renal damage and proceed to ESRD more frequently than their adult counterparts ${ }^{(4)}$.

The balance between proinflammatory and antiinflammatory cytokines influences the clinical manifestations in many inflammatory diseases such as SLE and rheumatoid arthritis (RA). These cytokines are mainly produced by helper $\mathrm{T}(\mathrm{Th})$ cells ${ }^{(7)}$.
Interleukin-17(IL-17) is a potent proinflammatory cytokine produced by the CD4+T-helper subset called

Th17 cells ${ }^{(8)}$. IL-17 promotes inflammation by inducing local chemokine and cytokine secretion (9) and is essential for the clearance of certain pathogens such as bacteria and fungi. Moreover, IL-17 has been linked to immune-mediated organ damage in the context of several autoimmune diseases ${ }^{(\mathbf{1 0})}$. The frequency of IL17 producing $\mathrm{T}$ cells is increased in the peripheral blood of adult SLE patients (11) and IL-17 production is abnormal in SLE patients. IL-17 levels are increased in adult SLE sera ${ }^{(8)}$.

The balance of Th17 and Th1 cell responses is also dysregulated in SLE patients and correlated with disease activity. IL-17 serves as a prognostic marker in SLE ${ }^{(8)}$. Vincent and his colleagues reported an important role for IL-17 in the pathogenesis of lupus nephritis ${ }^{(\mathbf{1 2})}$.

The present study was conducted to determine the serum IL-17 levels in pSLE patients and control groups and to evaluate the association between IL-17 and clinical manifestations, disease activity, laboratory findings, SLEDAI, and therapy.

\section{PATIENTS AND METHODS}

A case-control study was conducted at Pediatric Nephrology Units, and the Outpatient Nephrology Clinic of Children Hospital, Zagazig University. It was carried out over two years from September 2017 to 
August 2019. The study was performed on 60 children and they were classified into two groups; group ${ }_{1}$ was 30 children with SLE (5 males and 25 females). Their ages ranged from 10 to 15 years. Group 2 was 30 apparently healthy children.

\section{Ethical consideration:}

Approval for performing the study was obtained from Pediatrics Departments, Zagazig University Hospitals after taking Institutional Review Board (IRB) approval, and also informed written consent was taken from patients and/or their caregivers. This Work was performed according to the code of Ethics of the World Medical Association (Declaration of Helsinki) for studies involving humans. Inclusion criteria: Children with SLE had an age ranged from 5 to 18 years old. All pSLE cases were newly diagnosed. They were treated and followed at the Nephrology Unit of pediatrics and Outpatient Nephrology Clinic at Children Hospital. Disease activity was evaluated according to the SLE Disease Activity Index (SLEDAI) score (List of 24 items, 16 of which are clinical items and eight are laboratory results, scored based on whether these manifestations are present or absent in the previous 10 days, the weighted organ manifestations are then summed into a final score, which can range from zero to 105 and finally score was classified mild, moderate and severe).

The Control group from school children had no history of fever, rash, or joint pain at the time of examination and sampling; their antinuclear antibody and dsDNA antibody profile was negative.

Exclusion criteria: Patients with overlap syndrome were excluded from the study. Overlap syndrome is a medical condition which shares features of at least two more widely recognized disorders such as overlapping connective tissue disorders in rheumatology

\section{Methods:}

Each patient was subjected to complete history and full medication history taking with particular emphasis on age, sex, onset of disease, duration of disease before diagnosis, initial clinical presentation, full clinical examination was done for all patients (weight, length, blood pressure, malar rash, oral ulcer, photosensitivity, alopecia, seizure, psychosis, arthritis, pleural effusion, pericardial effusion, peritonitis, ascites, anemia, bleeding, petechiae purpura splenomegaly, lymphadenopathy, hepatomegaly, and fever).

Investigation: Routine laboratory investigation as Complete blood picture (CBC), Erythrocyte sedimentation rate (ESR), Urine analysis, kidney function tests (serum creatinine, blood urea nitrogen, albumin). 24-hour urinary protein, antinuclear antibody (ANA), anti-DNA, renal biopsy, Complement fractions $\mathrm{C} 3$ and $\mathrm{C} 4$, serum level of IL-17 by solid-phase sandwich ELISA.

\section{Test principle}

The kit uses a double-antibody sandwich enzymelinked immunosorbent assay (ELISA) to assay the level of Human Interleukin 17(IL-17) in samples. Add Interleukin 17(IL-17)to monoclonal antibody Enzyme well which is pre-coated with Human Interleukin 17(IL17) monoclonal antibody, incubation; then, add Interleukin 17(IL-17)antibodies labeled with biotin, and combined with Streptavidin-HRP to form an immune complex; then carry out. incubation and washing again to remove the uncombined enzyme. Then add Chromogen Solution A, B, the color of the liquid changes into the blue, And at the effect of acid, the color finally becomes yellow. The chroma of color and the concentration of the Human Substance Interleukin 17(IL-17) of the sample were positively correlated.

\section{Statistical analysis}

The collected data were computerized and statistically analyzed using the SPSS program (Statistical Package for Social Science) version 24.0 (SPSS Inc., 2007). For the statistical calculations, data coding was done. The test results were considered significant when the P-value $\leq 0.05$, highly significant when $\mathrm{P}$-value $\leq 0.01$, and non-significant when P-value $>0.05$. All $\mathrm{p}$ values are two-tailed. (Levesque, 2007).

\section{RESULTS}

This study showed a highest percentage of malar rash $(86.7 \%)$ followed by photosensitivity $(80 \%)$ and renal manifestation was presented by $73.3 \%$. The two least presentations in the case group were neurological manifestation (13.3\%) followed by bleeding and ecchymosis were (6.7\%). Table (1)

This study showed that all SLE cases were treated by methylprednisolone and prednisolone as specific therapy and calcium, vitamin D, and antacid as supportive therapy. Ninety percent were treated by Hydroxychloroquine, $80 \%$ of cases were treated by cyclophosphamide. Some cases and Mycophenolate mofetil $(6.6 \%)$. Non-responding cases for the previous line of treatment were treated by intravenous immunoglobulin and plasmapheresis (6.6\%). Antiplatelet therapy and Low MW Heparin were used for treatment anti-cardiolipin and lupus anti-coagulant positive (6.6\%). Table (2)

There was a highly statistically significant increase in the Il-17 level in the case group than the control. Table (3).

There was a significant association between IL-17 and body swelling (puffiness of eyes and lower limb with abdominal swelling) and dark-colored urine. There was a significant association between 17 with anemia and renal manifestation. Table (4)

The bar chart showed a comparison score of SLEAI and IL17, $40.0 \%$ of cases with IL-17 $\geq 170$ had severe SLEDAI. Figure (1)

That there was a highly significant positive correlation between IL-17 level and SLEDAI score. Figure (2): There was a significant association between 
IL-17 level and SLEDAI. $40.0 \%$ of cases with IL$17 \geq 170$ had high SLEDAI. Table (5)

Table (1): Frequencies of initial clinical manifestations for the case group

\begin{tabular}{|l|c|}
\hline \multicolumn{1}{|c|}{ Variables } & $\begin{array}{c}\text { Case Group } \\
(\mathrm{n}=30)\end{array}$ \\
\hline Malar rash & $2686.7 \%$ \\
\hline Photosensitivity & $2480.0 \%$ \\
\hline Renal manifestations & $2273.3 \%$ \\
\hline Oral ulcers & $1756.7 \%$ \\
\hline Serositis & $1653.3 \%$ \\
\hline Arthritis & $1473.3 \%$ \\
\hline Hematological manifestations: & $2275.0 \%$ \\
Anemia & $1033.3 \%$ \\
Infection & $26.7 \%$ \\
Bleeding/ecchymosis & $413.3 \%$ \\
\hline Neurological manifestations & 20 \\
\hline SLEDAI score: & $18-38$ \\
Median & \\
Range &
\end{tabular}

Table (2): Drug therapy in the case group during the study period

\begin{tabular}{|l|c|c|}
\hline \multirow{2}{*}{ Variables } & $\begin{array}{c}\text { Case Group } \\
(\mathbf{n}=\mathbf{3 0})\end{array}$ \\
\cline { 2 - 3 } Specific therapy & No. & $\%$ \\
\hline \multicolumn{2}{|c|}{} \\
\hline Methylprednisolone & 30 & 100 \\
\hline Prednisolone & 30 & 100 \\
\hline Hydroxychloroquine & 27 & 90 \\
\hline Cyclophosphamide & 24 & 80 \\
\hline Mycophenolate mofetil & 6 & 20 \\
\hline Intravenous immunoglobulin & 2 & 6.6 \\
\hline Plasmapheresis & 2 & 6.6 \\
\hline \multicolumn{2}{|c|}{ Supportive therapy } & \\
\hline Calcium & 30 & 100 \\
\hline Vitamin D & 30 & 100 \\
\hline Antacid & 30 & 100 \\
\hline Antihypertensive therapy & 14 & 46.6 \\
\hline $\begin{array}{l}\text { Antiplatelet therapy and Low } \\
\text { MW Heparin }\end{array}$ & 2 & 6.6 \\
\hline Albumin & 12 & 40 \\
\hline \hline
\end{tabular}

Table (3): Level of IL-17 in studied groups

\begin{tabular}{||c|c|c|c|c|}
\hline IL-17 $(\mathbf{p g} / \mathbf{m l})$ & Case Group $(\mathbf{n = 3 0})$ & $\begin{array}{c}\text { Control Group } \\
(\mathbf{n = 3 0})\end{array}$ & T & P-value \\
\hline X \pm SD & $188.5 \pm 44.6$ & $73.7 \pm 10.5$ & \multirow{2}{*}{10.0} & \multirow{2}{*}{$\mathbf{0 . 0 0 0 * *}$} \\
\hline Median & 170.0 & 70.0 & & \\
\hline Range & $89.3-359.0$ & $60.5-92.0$ & \\
\hline
\end{tabular}

** High statistical significance.

Table (4): Association between IL-17 level and initial complaints of case group.

\begin{tabular}{|c|c|c|c|c|c|c|}
\hline \multirow{3}{*}{ Variables } & \multicolumn{4}{|c|}{ IL-17 levels } & \multirow{3}{*}{$X^{2}$} & \multirow{3}{*}{ P-value } \\
\hline & \multicolumn{2}{|c|}{$<170 \mathrm{pg} / \mathrm{ml}$} & \multicolumn{2}{|c|}{$\geq 170 \mathrm{pg} / \mathrm{ml}$} & & \\
\hline & No. & $\%$ & No. & $\%$ & & \\
\hline Fever $(n=16)$ & 5 & 31.2 & 11 & 8.8 & 1.1 & 0.3 \\
\hline Facial rash $(n=13)$ & 7 & 53.8 & 6 & 46.2 & Fisher & $\overline{0.3}$ \\
\hline Joint pain \& swelling $(n=12)$ & 6 & 50.0 & 6 & 50.0 & Fisher & 0.1 \\
\hline Abdominal pain $(n=10)$ & 4 & 40.0 & 6 & 60.0 & Fisher & 0.99 \\
\hline Headache(=9) & 5 & 55.6 & 4 & 44.4 & Fisher & 0.4 \\
\hline Hair falling $(=9)$ & 4 & 44.4 & 5 & 55.6 & Fisher & 0.3 \\
\hline Oral ulcers $(=8)$ & 4 & 50.0 & 4 & 50.0 & fisher & 0.1 \\
\hline Pallor $(n=8)$ & 2 & 25 & 6 & 75 & Fisher & 0.4 \\
\hline Puffiness of eyes (n=8) & 1 & 12.50 & 7 & 87.5 & Fisher & $0.02 *$ \\
\hline Swelling of legs-Abd. Swelling $(n=8)$ & 1 & 12.50 & 7 & 87.5 & Fisher & $0.02 *$ \\
\hline Decreased urine volume $(n=8)$ & 3 & 37.5 & 5 & 62.5 & Fisher & 0.5 \\
\hline Dark colored urine $(n=7)$ & 1 & 14.2 & 6 & 85.8 & Fisher & 0.03* \\
\hline Photosensitivity $(\mathrm{n}=6)$ & 4 & 66.6 & 2 & 33.4 & Fisher & 0.1 \\
\hline Bleeding $(n=2)$ & 1 & 50.0 & 1 & 50.0 & Fisher & 0.3 \\
\hline Vomiting $(n=2)$ & 1 & 50.0 & 1 & 50.0 & fisher & 0.3 \\
\hline
\end{tabular}




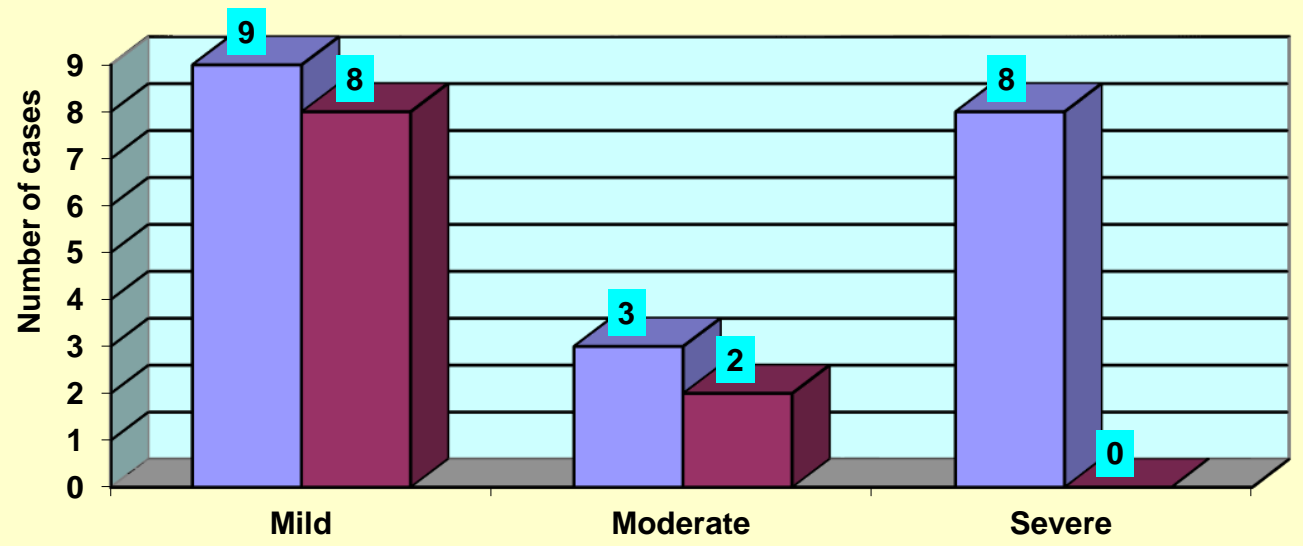

Figure (1): Bar chart showing the comparison between SLE cases with IL-17 above and below 170 as regards SLEDAI

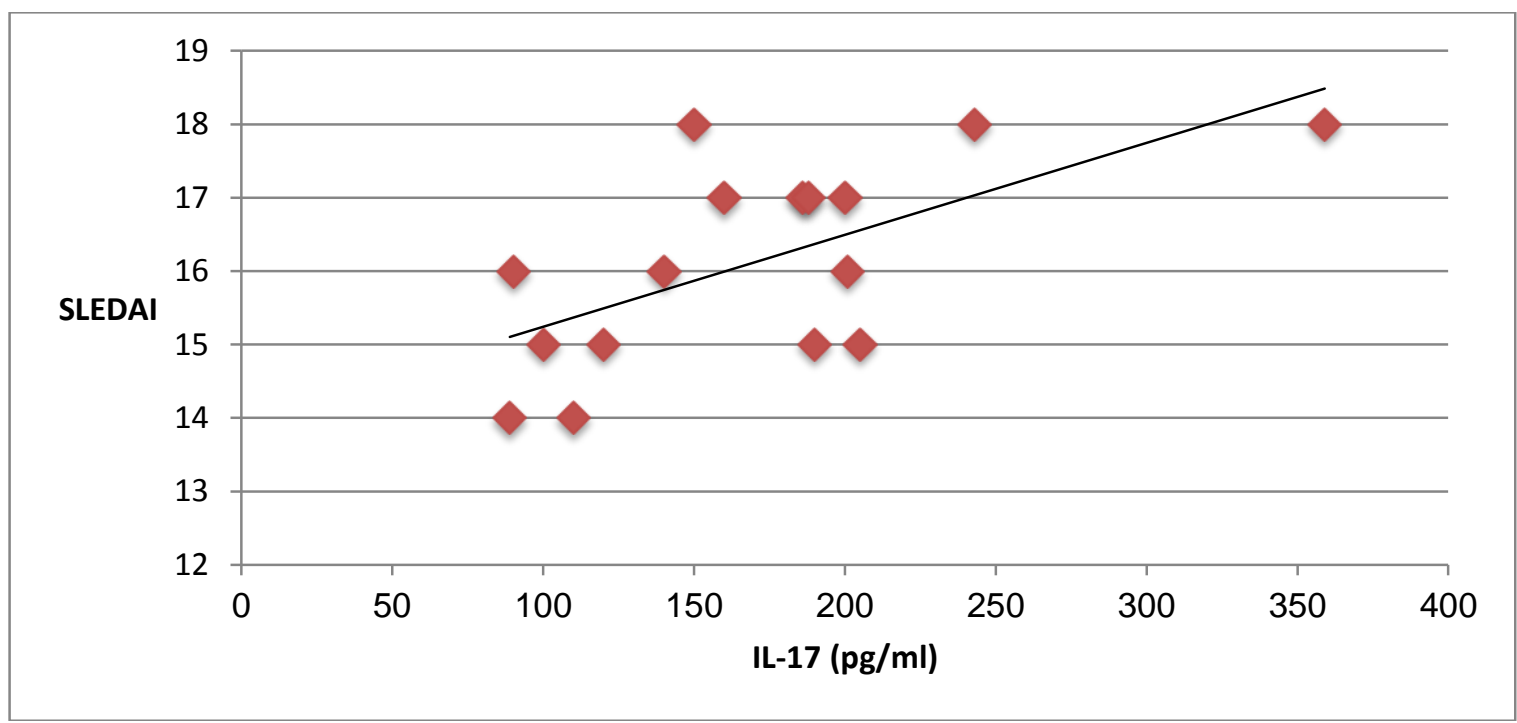

Figure (2): Correlation between IL-17 level and SLEDAI of the case group

Table (5): Association between IL-17 level and initial clinical manifestations of case group.

\begin{tabular}{|c|c|c|c|c|c|c|}
\hline \multirow{3}{*}{ Variables } & \multicolumn{4}{|c|}{ IL-17 levels } & \multirow{3}{*}{$X^{2}$} & \multirow{3}{*}{ P-value } \\
\hline & \multicolumn{2}{|c|}{$\begin{array}{c}<170 \mathrm{pg} / \mathrm{ml} \\
(\mathrm{n}=10)\end{array}$} & \multicolumn{2}{|c|}{$\begin{array}{c}\geq 170 \mathrm{pg} / \mathrm{ml} \\
(\mathrm{n}=20)\end{array}$} & & \\
\hline & No. & $\%$ & No. & $\%$ & & \\
\hline Malar rash (26) & 10 & 100 & 16 & 80 & Fisher & 0.4 \\
\hline Photosensitivity(24) & 8 & 80 & 16 & 80 & Fisher & 0.07 \\
\hline Renal manifestations (22) & 2 & 20 & 20 & 100 & Fisher & $0.001 *$ \\
\hline Oral ulcers(17) & 9 & 90 & 8 & 40 & Fisher & 0.6 \\
\hline Serositis(16) & 9 & 90 & 7 & 35 & Fisher & 0.6 \\
\hline Arthritis(14) & 8 & 80 & 6 & 30 & Fisher & 0.6 \\
\hline Neurological manifestations(4) & 2 & 20 & 2 & 10 & Fisher & 0.2 \\
\hline Hematological manifestations: & & & & & & \\
\hline Anemia (22) & 4 & 40 & 18 & 90 & fisher & 0.003* \\
\hline Infection(10) & 4 & 40 & 6 & 30 & fisher & 0.4 \\
\hline Bleeding/ecchymosis (2) & 1 & 10 & 1 & 5 & fisher & 0.2 \\
\hline
\end{tabular}




\section{DISCUSSION}

Lupus nephritis is one of the major causes of morbidity in SLE and there has been significant effort and encouragement in understanding the pathogenesis and renal histologic classification. LN is a severe form of SLE organ involvement that may progress to endstage renal failure unless properly treated ${ }^{(\mathbf{1 3})}$. Many cytokines are also involved and contribute to both onset and progression of renal pathology ${ }^{(\mathbf{1 4})}$.

While we still do not possess a validated biomarker for lupus nephritis, as conventional serum biomarkers have not proved to be an appropriate gauge of lupus nephritis. SLE is a systemic disease, serum biomarkers may indeed be appropriate monitoring and diagnostic tools for systemic disease activity. Concerning lupus nephritis, however, urinary biomarkers may be more specific for kidney damage than serum biomarkers, particularly in SLE patients with active systemic disease ${ }^{(\mathbf{1 5})}$.

Even though some reports are indicating that IL17(serum and urinary) and Th17 cells play important roles in the pathogenesis of $\mathrm{LN}^{\left({ }^{(16)}\right.}$.

In our study, the malar rash was a main presenting symptom (having a percentage of $(86.7 \%)$. Disagree with many results in all over the world (Thailand study was done by Pattaragarn et al. ${ }^{(17)}$ Philippinian study done by Hamijoyo et al. ${ }^{(18)}$, and Egyptian study done by Elmougy et al. ${ }^{(19)}$ and Youssef et al. ${ }^{(20)}$. Most of this study had a lower presentation than our current study.

The current study showed that serositis was represented by $53.3 \%$. On the other hand, Hammad $\boldsymbol{e t}$ al. (21) who reported serositis was the least frequent clinical presentation (8\%).

The frequency of arthritis in our study was 46.6\% compared to other studies involving Egyptian one carried out by Salah et al. ${ }^{(22)}$, Youseff et $\boldsymbol{a l} .{ }^{(20)}$ and Hammad et al. ${ }^{(21)}$ Indian byGulay and Das ${ }^{(23)}$ and European children Costagliola et al., (24) but less common than in Arab Habib and Saliba ${ }^{(25)}$ and Alsaeid et al. ${ }^{(26)}$.

Our patients had fewer CNS manifestations $13.3 \%$; this result is supported by Youssef et al. ${ }^{(20)}$ and Hammad et al. ${ }^{(21)}$ in Egypt (11.5\%) and Alsaeid et al. ${ }^{(26)}$ in Kuwaiti who reported a low frequency of neuropsychiatric abnormalities in children with SLE. However, reports from Saudi Arabia Bahabri et al. (27), Western countries Bruns and Meyer ${ }^{(28)}$, and the Philippine study Gulay and Dans ${ }^{(23)}$ were Pointed to a higher frequency of CNS abnormalities.

The renal manifestations of $\mathrm{LN}$ patients in the current study were presented $(73.3 \%)$ in the form proteinuria $(80.0 \%)$ hematuria $(30 \%)$ and urinary casts $(13.3 \%)$, hypertension (30\%) and without impairment of renal function at the time of study for SLE cases.
Different of studies were carried in Egypt Hammad $\boldsymbol{e t}$ al. (21) found that renal manifestation was the most frequent manifestation (81\%); Bakr et al. ${ }^{(29)}$ and Elmougy et al. ${ }^{\left({ }^{19)}\right)}$ were reported proteinuria $(83.3 \%)$, hematuria (71.5\%), hypertension (35.7\%) and elevated creatinine elevated serum creatinine (16.7\%). Higher values with Youssef et al. ${ }^{(20)}$, proteinuria (100\%), and hematuria (84.6\%) without impairment in kidney function, like Hoffman et al. ${ }^{(30)}$. It was higher than the $41 \%$ and $24.3 \%$, respectively, found in a study carried out by Gulay and Dans ${ }^{(23)}$. Hypertension was (76.9\%) in Youssef et al. ${ }^{(20)}$ higher than our results but $40 \%$ found in other studies Perfumo and Martini ${ }^{(31)}$ and Hersh et al. ${ }^{(32)}$.

The current study showed that hematological involvement was observed in 10 patients at disease onset (pallor in 8 cases $(26.6 \%$ ), and bleeding and petechiae in 2 cases $(6.6 \%)$ ).

The frequency of anemia in our study was $73.3 \%$ which less than in comparison to Youssef $\boldsymbol{e t}$ al. (20) $(80.8 \%)$, which is more than other studies performed by Bakr et al. ${ }^{(29)}$ (51\%), the Kuwaiti study (20\%) carried out by AlSaeid et al. ${ }^{\text {(26) }}$.

Our study showed all SLE cases were treated by methylprednisolone and prednisolone as specific therapy. Ninety percent were treated by hydroxychloroquine, $80 \%$ of cases were treated by cyclophosphamide whose renal manifestation. Some cases were treated by mycophenolate mofetil (20\% for resistant cases with renal manifestation). Nonresponding cases for the previous line of treatment were treated by intravenous immunoglobulin and plasmapheresis (6.6\%). Low MW Heparin and antiplatelet therapy and were used for treatment anticardiolipin and lupus anti-coagulant positive (6.6\%).

Our study showed a highly significant increase in the Il-17 level in the case group than the controls. In agreement with another study in Egypt ${ }^{(21)}$. Peliçari $\boldsymbol{e t}$ al. ${ }^{(33)}$ and his collages also reported that increased levels of IL-17 can be considered as a risk factor for the development of SLE in children.

We detected higher IL-17 levels in SLE patients with nephritis. Our study showed a significant association between IL-17 and different renal symptoms as body swelling (puffiness of eyes and lower limb with abdominal swelling) and dark-colored urine as initial complaint and a highly significant increase in the renal manifestation of SLE cases with high $\mathrm{IL}-17 \geq 170$. Our data are in agreement with previous studies suggesting that IL-17 could contribute to the pathogenesis of kidney involvement in SLE patients ${ }^{(33,34,35)}$. Support for the pathogenic role of IL17 derives from the fact that IL-17-producing T cells have been observed in the kidneys of patients with lupus nephritis ${ }^{(33)}$. IL-17 is mostly produced by $\mathrm{CD} 4^{+}$, $\mathrm{CD}^{+}, \mathrm{CD} 4^{-} / \mathrm{CD}^{-}, \gamma / \delta \mathrm{T}$ cells, and Th17 cells. In 
human lupus nephritis, IL-17-expressing $\mathrm{T}$ cells migrate to the kidneys and contribute to inflammatory processes. However, other studies for SLE children did not demonstrate an association between IL-17 and nephritis. These findings may be due to the smaller number of patients included or to different disease duration ${ }^{(34,35)}$.

The current study showed a significant association between IL-17 level and SLEDAI scores. $40.0 \%$ of cases with IL-17 $\geq 170$ had high SLEDAI and a highly significant positive correlation between IL-17 level and SLEDAI. Forty percent of cases had severe SLEDAI and IL-17 above $170 \mathrm{pg} / \mathrm{ml}$.

The association between IL-17 levels and disease activity remains controversial ${ }^{(35)}$. Although some studies observed a positive association, our study recorded a significant association between IL-17 level and SLEDAI. I agree with disagreeing with Peliçari $\boldsymbol{e t}$ al. ${ }^{(33)}$. The difference can be explained by the different genetic backgrounds, different disease duration heterogeneity of clinical and laboratory manifestations in SLE and different sample size (we had a larger number of patients), and variations in the principle and sensitivity of the ELISA tests.

\section{CONCLUSION}

Serum IL-17A is elevated $>170$ in pSLE patients with lupus nephritis. IL-17 seems to have a possible role in the pathogenesis of lupus nephritis.

\section{RECOMMENDATION}

Longitudinal studies with regular monitoring of serum and urine IL-17 will be necessary to determine the sensitivity and specificity of IL-17 as a predictor marker as a diagnostic and prognostic marker for lupus nephritis in pSLE.

\section{REFERENCES}

1. Rahman A, Isenberg D (2008): Systemic lupus erythematosus. N Engl J Med., 358:929-39.

2. Levy DM, Kamphuis S (2012): Systemic Lupus Erythematosus in Children and Adolescents. Pediatric Clinics of North America, 59(2): 345-64.

3. Watson L, Leone V, Pilkington C et al. (2012): Disease activity, severity, and damage in the UK Juvenile-Onset Systemic Lupus Erythematosus Cohort. Arthritis Rheum., 64: 2356-65.

4. Mina R, Brunner H (2010): Pediatric lupus - are there differences in presentation, genetics, response to therapy, and damage accrual compared with adult lupus? Rheum Dis Clin North Am., 36:53-80.

5. Ardoin S, Schanberg L (2012): Pediatric rheumatic disease: lessons from SLE: children are not little adults. Nat Rev Rheumatol., 8: 444-5.

6. Maroz N, Segal M (2013): Lupus nephritis and end-stage kidney disease. Am J Med Sci., 346: 319-23.
7. Steinman L (2007): A brief history of TH17, the first major revision in the TH1/TH2 hypothesis of $\mathrm{T}$ cellmediated tissue damage. Nature Medicine, 13(2): 139-45.

8. Chen D, Chen Y, Hung W et al. (2012): The potential role of Th-17cell and Th-17 related cytokine in the pathogenesis of lupus nephritis. SAGE Journals, 2012; 317.

9. Crispín J, Tsokos G (2010): IL-17 in Systemic Lupus Erythematosus. J Biomed Biotechnol., 22(5):499-503.

10. Nalbandian A, Crispín J, Tsokos G (2009): Interleukin17 and systemic lupus erythematosus: current concepts. Clin and Exp Immunology, 157 (2):209-15.

11. Shah K, Lee W, Lee S et al. (2010): Dysregulated balance of Th17 and Th1 cells in systemic lupus erythematosus. Arthritis Res Ther., 12(2): 53-58.

12. Vincent F, Northcott M, Hoi A et al. (2013): Clinical associations of serum interleukin-17 in systemic lupus erythematosus. Arthritis Research \& Therapy, 15(4), 23-9.

13. Zampeli E, Klinman D, Gershwin $M$ et al. (2017): A comprehensive evaluation for the treatment of lupus nephritis. J Autoimmun., 78:1-10.

14. Zickert A, Amoudruz P, Sundström Y et al. (2015): IL17 and IL-23 in lupus nephritis - association to histopathology and response to treatment. BMC Immunology, 16:7-9.

15. Thomas J, Blanco I, Putterman C (2011): Urinary Biomarkers in Lupus Nephritis. Clin Rev Allergy Immunol., 40(3): 138-150.

16. Koga T, Ichinose $K$, Tsokos $\mathbf{G}$ (2017): $T$ cells and IL-17 in lupus nephritis. Clin Immunol., 185: 95-9.

17. Pattaragarn A, Sumboonnanonda A, Parichatikanond P et al. (2005): Systemic lupus erythematosus in Thai children: Clinicopathologic findings and outcome in 82 patients. J Med Assoc Thai., 88(8):232-41.

18. Hamijoyo L, Bernal C, Navarra S (2009): Comparison of the clinical presentation of childhood/ adolescent-onset with adult-onset systemic lupus erythematosus. Philippine Rheumatology Association, 16 ${ }^{\text {th }}$ Annual Meeting; 2009. Lupus nephritis in Lebanon. Lupus, 10:378-81.

19. Elmougy A, Sarhan A, Hammad A (2015): Erratum to: Lupus nephritis in Egyptian children: a 16-year experience. J Nephrol., 28(1):131.

20. Youssef D, Tawfek D, Mohammed A et al. (2015): Pediatric systemic lupus erythematosus in a single nephrology unit. Saudi J Kidney Dis and Transplantation, 26(2): $314-926$.

21. Hammad A, Osman E, Mosaad Y et al. (2017): Serum interleukin-17 in Egyptian children with systemic lupus erythematosus: is it related to pulmonary affection. Lupus, 26(4): 388-95.

22. Salah S, Lotfy H, Sabry S et al. (2009): Systemic lupus erythematosus in Egyptian children. Rheumatol Int., 29:1463-8.

23. Gulay C, Dans L (2011): Clinical presentations and outcomes of Filipino juvenile systemic lupus erythematosus. Pediatric Rheumatol Online J., 9:7-12.

24. Costagliola G, Mosca M, Migliorini $P$ et al. (2018): Pediatric Systemic Lupus Erythematosus: Learning From Longer Follow Up to Adulthood Front Pediatr., 6: 144-48.

25. Habib G, Saliba W (2002): Systemic lupus erythematosus among Arabs. Isr Med Assoc J., 4:690-3. 
26. Alsaeid K, Kamal H, Haider M et al. (2004): Systemic lupus erythematosus in Kuwaiti children: Organs involvement and serological findings. Lupus, 13: 613-7.

27. Bahabri S, Sabban E, Al Rashed A et al. (1997): Juvenile systemic lupus erythematosus in 60 Saudi children. Ann Saudi Med., 17: 612-5.

28. Bruns A, Meyer O (2006): Neuropsychiatric manifestations of systemic lupus erythematosus. Joint Bone Spine, 73: 639-45.

29. Bakr A (2005): Epidemiology treatment and outcome of childhood systemic lupus erythematosus in Egypt. Pediatr Nephrol., 20 (8):1081-6.

30. Hoffman I, Lauwerys B, De Keyser F et al. (2009): Juvenile-onset systemic lupus erythematosus: Different clinical and serological pattern than adult-onset systemic lupus erythematosus. Ann Rheum Dis., 68:412-5.

31. Perfumo F, Martini A (2005): Lupus nephritis in children. Lupus, 14:83-8.
32. Hersh A, von Scheven E, Yazdany J et al. (2009): Differences in long-term disease activity and treatment of adult patients with childhood- and adult-onset systemic lupus erythematosus. Arthritis Rheum., 61:13-20.

33. Peliçari K, Sinicato N, Peres F et al. (2015): Serum interleukin-17 levels are associated with nephritis in childhood-onset systemic lupus erythematosus. Clinics (Sao Paulo), 70(5): 313-7.

34. Mok M, Wu H, Lo Y et al. (2010): The relation of interleukin 17 (IL-17) and IL-23 to Th1/Th2 cytokines and disease activity in systemic lupus erythematosus. J Rheumatol., 37(10):2046-52.

35. Rana A, Minz R, Aggarwal R et al. (2012): Gene expression of cytokines (TNF-alpha, IFN-gamma), serum profiles of IL-17 and IL-23 in pediatric systemic lupus erythematosus. Lupus, 21(10):1105-12. 